\title{
Interval timing with a beat frequency model connecting phase resetting and network response
}

\author{
Steven Dix ${ }^{1}$, Sorinel A Oprisan ${ }^{2 *}$, Catalin V Buhusi ${ }^{3}$ \\ From Nineteenth Annual Computational Neuroscience Meeting: CNS*2010 \\ San Antonio, TX, USA. 24-30 July 2010
}

Time perception is essential for normal physiological and behavioral responses, e.g., recalling stored data and planning future actions, and is deregulated in a series of neuropathologies, e.g., Parkinson's disease. It is commonly accepted that processing temporal information involves a neural mechanism mimicking a stopwatch such as in the Scalar Expectancy Theory [1]. Organisms have multiple neural timing mechanisms that can span more than 10 orders of magnitude form circadian (24 hours) to interval (minutes) to millisecond timing [2]. Our focus is on modeling neural networks responsible for interval timing. In designing any computational model of interval timing, we must acknowledge an experimentally observed fact: the plot of mean response time versus the standard deviation of the distribution for different timing intervals is linear (scalar property). The established paradigm assumes an internal clock model in which pulses that are emitted regularly by a pacemaker are temporarily stored in an accumulator. At the time of reward or feedback, the number of pulses stored in the accumulator is transferred to the reference memory for future use. During "peak-interval procedure" (in which the participants are asked to reproduce the criterion interval) it is assumed that the internal clock is reset and pulses stored into accumulator until the current count match the memorized value stored during the training. The model we used assumes that timing is an emergent activity originated in the thalamo-cortico-striatal loops $[2,3]$. In this striatal beat-frequency (SBF) model, timing is based on the coincidental activation of medium spiny neurons in the basal ganglia by cortical neural oscillators [2,3]. The cortical

\footnotetext{
* Correspondence: oprisans@cofc.edu

${ }^{2}$ Physics and Astronomy Department, College of Charleston, Charleston, SC 29424, USA
}

oscillators are assumed to be synchronized at the onset of a trial, and to oscillate at a fixed frequency throughout the criterion interval. In our computational model, the oscillator block is composed of independent spiking neurons with intrinsic frequencies $f i$ which, due to varying factors (biological noise, background neural activity from other cortical areas, etc.), are randomly distributed according to a certain density probability function. The synchronization of cortical oscillations at trial onset and the experience-dependent changes in cortico-striatal transmission are thought to be modulated by the dopaminergic neurons in the substatia nigra pars compacta and ventral tegmental area. A memory block stores the criterion time value $c$ memorized during the training process. However, both storage and retrieval of criterion time to and from memory units is affected by biological context (brain state) and is modeled by randomly distributing criterion time according to a certain density probability function [3]. All timing models must connect the objective time with the subjective time. The subjective time is strongly influenced by brain's state through neuromodulators. For example, dopamine advances the response whereas achetylcholine can induce a delay of the response in a fixed interval experiment. The mechanism involved could be through a direct change in firing frequency due to neurotransmitter action and it was modeled as a separate clock block. The decision block is an essential component of any timing mechanism that relates internal perception of time intervals with external action. A straightforward mechanism of implementing decision-making uses a set of synaptic weights derived from the memorized criterion time [3]. The weights computed from the memorized criterion constitute the reference vector of weighs. The decision block estimates the projection of the current weights on 
the vector of weight reference weight. The model correctly reproduced measured behavioral data.

\section{Acknowledgements}

This work was supported by a Summer Undergraduate Research with Faculty fellowship to SD, a College of Charleston Research and Development grant to SAO, and NIH grant MH073057 to CVB.

\section{Author details}

'Computer Science Department, College of Charleston, Charleston, SC 29424, USA. ${ }^{2}$ Physics and Astronomy Department, College of Charleston, Charleston, SC 29424, USA. ${ }^{3}$ Neurosciences Department, Medical University of South

Carolina, Charleston, SC 29425, USA.

Published: 20 July 2010

\section{References}

1. Church RM: Properties of an internal clock. Ann N Y Acad Sci 1984, 423:566-582.

2. Buhusi $\mathrm{CV}$, Meck WH: What makes us tick? Functional and neural mechanisms of interval timing. Nat. Rev. Neurosci 2005, 6:755-765.

3. Matell MS, Meck WH: Cortico-striatal circuits and interval timing: coincidence detection of oscillatory processes. Brain Res Cogn Brain Res 2004, 21(2):139-170.

doi:10.1186/1471-2202-11-S1-P95

Cite this article as: Dix et al:: Interval timing with a beat frequency model connecting phase resetting and network response. $B M C$

Neuroscience 2010 11(Suppl 1):P95.

\section{Submit your next manuscript to BioMed Central} and take full advantage of:

- Convenient online submission

- Thorough peer review

- No space constraints or color figure charges

- Immediate publication on acceptance

- Inclusion in PubMed, CAS, Scopus and Google Scholar

- Research which is freely available for redistribution

Submit your manuscript at www.biomedcentral.com/submit 